\title{
Evaluación de la Enfermedad periodontal como factor de riesgo para el parto prematuro y bajo peso al nacer, en las embarazadas que asisten al servicio de Gineco-obstetricia del Hospital Amistad México-Nicaragua del municipio de Ticuantepe, en el primer semestre del año 2016 \\ MSc. Rubén Alexander Martínez González Docente investigador UNAN-MANAGUA, Facultad de Ciencias Médicas byr2093@hotmail.com
}

Palabras clave: Periodontitis, Complicaciones Perinatales, Bajo Peso al Nacer, Parto Pretérmino, Recién Nacidos de bajo peso al nacer.

\section{RESUMEN}

$\mathrm{E}$ I Parto Pretérmino (PP) y Bajo Peso al Nacer (BPN) es un problema serio en los países desarrollados y en los países en vías de desarrollo, pues causa el 35\% de las muertes neonatales y es la segunda causa de muerte solo sobrepasado por la Neumonía. En Nicaragua, se calcula que para el año 1990 al 2010, la tasa de nacimiento prematuros era del 9.3\% por cada 100 nacidos vivos. Este fenómeno ha sido vinculado a varios procesos infecciosos que podrían causar una respuesta inflamatoria en el líquido amniótico y causar PP y BPN; de estas infecciones, la Enfermedad Periodontal (EP) no está exenta. El objetivo de este estudio es evaluar la periodontitis como factor de riesgo para causar parto pretérmino y bajo peso al nacer. Para el estudio se examinó a 29 gestantes, quienes fueron ordenadas en cuatro grupos experimentales según el tipo de EP. Se realizaron dos exámenes clínicos; el primero, fue previo al tratamiento dental y el segundo examen, después del tratamiento y antes de cumplido las 35 semanas gestacional. Los resultados muestran que, el $89.7 \%$ de las pacientes presentó periodontitis moderada y $10.3 \%$ periodontitis leve. El 27.6\% presentó PP y el 20.7\%, BPN. No se encontró relación estadística significativa entre la EP ni el tipo de periodontitis sobre el PP $(\boldsymbol{p}=\mathbf{0 . 2 4})$ ni sobre el BPN $(\boldsymbol{p}=\mathbf{0 . 8 1})$. Estos resultados indican que la enfermedad periodontal no es un factor causal ni de riesgo para el PP y BPN en las embarazadas que asisten al hospital Amistad México-Nicaragua.

\section{INTRODUCCIÓN}

El parto prematuro y bajo peso al nacer son patologías que se presentan en la mayoría de los casos de forma simultánea y causan el 35\% de las muertes neonatales a nivel mundial, convirtiéndose en la segunda causa de muerte de los neonatos solo sobrepasado por la neumonía. El parto pretérmino es definido como el nacimiento del producto antes de las 37 semanas de gestación o 259 días y se describe el bajo peso al nacer a todo aquel niño que nace pesando menos de 2500 gr (Beck, y otros, 2010). 
Por otro lado, la enfermedad periodontal es una de las enfermedades más comunes y la mayor causa de pérdida dentaria y edentulismo en pacientes adultos. Es una patología con numerosos factores que intervienen en su desarrollo como factores microbiológicos, factores ambientales y sistémicos como el embarazo, que debido a los cambios hormonales que provoca, induce al empeoramiento de la respuesta inflamatoria en el periodonto. Beck y otros (2010) afirman que la enfermedad periodontal es posible causante de neonatos nacidos antes de término y con bajo peso al nacer. Esta asociación se atribuye a la invasión de las bacterias peridontopatógenas que viajan por vía hematógena hasta el líquido amniótico donde provocan una respuesta inflamatoria.

Con el objetivo de evaluar la enfermedad periodontal como factor de riesgo para el parto prematuro y bajo peso al nacer, en las embarazadas que asisten al servicio de Gineco-obstetricia del Hospital Amistad México - Nicaragua del municipio de Ticuantepe, en el primer semestre del año 2016, se necesitó el diseño y ejecución de un estudio cuasiexperimental, observacional y analítico.

Se diseñaron cuatro tipos de tratamientos y 10 repeticiones estadísticas en cada uno de ellos y se puso a prueba la hipótesis de investigación: La enfermedad periodontal podría ser un factor de riesgo para el parto prematuro y bajo peso al nacer, en las embarazadas que asisten al servicio de Gineco-obstetricia del Hospital Amistad México - Nicaragua en el primer semestre del año 2016.

Los resultados de este estudio son de gran valor para el hospital y el Sistema Nacional de Salud, porque se demostró la no relación de la enfermedad periodontal como un factor de riesgo para el parto pretérmino y bajo peso al nacer, de manera que el Sistema de Salud refuerce y haga prioridad la atención y manejo de la salud buco-dental de las gestantes en pro de garantizar y mejorar el estado de salud periodontal de las gestantes. Además, de ser un aporte a la producción científica del país.

\section{MATERIAL Y MÉTODO}

Este estudio prospectivo, cuasiexperimental y analítico, fue realizado en la Clínica Odontológica del Hospital Amistad México-Nicaragua del municipio de Ticuantepe, Managua, Nicaragua en colaboración con el servicio de Gineco-obstetricia, en el periodo comprendido entre enero a junio del año 2016. Se estudió un total de 29 mujeres embarazadas entre 18 a 35 años de edad, que no presentaran ningún tipo de adicción a drogas, alcohol o cigarrillo, que presentaran más de 20 dientes en la boca y que dieran su consentimiento para formar parte del estudio. Se distribuyeron en 3 diferentes grupos experimentales, diseñados a partir del diagnóstico de enfermedad periodontal de cada paciente. Los datos se recolectaron a través de inspección clínica, haciendo uso de periodontograma tomado de Ramseier (2010) y modificado para ser usado en formato no digital (impreso). Se les anexó apartados donde se incluyen los datos socioeconómicos de los pacientes, diagnóstico periodontal y casillas referentes a la presencia de parto prematuro y bajo peso al nacer. Se agregó además, el índice de sangrado gingival de Mühlrmann.

Se realizó el chequeo general de la cavidad oral y posteriormente, se utilizó una sonda periodontal de William y periodontograma, evaluándose nivel de inserción, presencia de bolsa periodontal, margen gingival y sangrado al sondaje. Se realizaron tres mediciones (distal, medial y mesial) en cada cara de cada pieza (vestibular y lingual / palatino). Primeramente, se inició introduciendo la sonda en el surco gingival de las porciones distales de cada pieza por su cara vestibular (hasta progresar a la zona mesial), se obtuvo el valor desde el margen gingival hasta el fondo del surco, 
teniendo en cuenta una medición mayor a tres milímetros, esto fue considerado como bolsa periodontal, luego se retiró la sonda haciendo un ligero contacto con el diente hasta localizar la unión amelo-cementaria y siempre tomado de referencia el margen gingival, se anotó en la ficha la medición del margen (pudiendo ser negativa o positiva).

Para obtener la medida de pérdida de inserción clínica se restó a la profundidad de sondeo la medición del margen gingival o se sumó cuando los valores eran negativos. La medición de sangrado se hizo luego de realizar a cada diente los dos procedimientos antes expuestos. Para la medición de la movilidad dental se utilizó el mango del espejo y el dedo índice de la mano izquierda, aplicando fuerzas en sentido vestíbulo lingual / palatino para cada diente. Una vez obtenidas las mediciones, el criterio diagnóstico para cada caso se definió siguiendo el criterio clínico expuesto por Armitage (1999).

\section{RESULTADOS}

Se registró un total de 29 embarazadas entre los 18 y 35 años de edad con una media de 23.5 años, de las cuales el $0.62 \%$ pertenece a la zona rural y el $38 \%$, a la zona urbana. El $17 \%$ tiene una escolaridad que llega a primaria; $14 \%$ del total de las pacientes estudiadas han obtenido el grado de profesional y el $0.69 \%$ han culminado la secundaria y obtenido el grado de bachiller.

Para valorar el estado de salud periodontal, se realizaron tablas de contingencia, las cuales muestran que, de los 29 casos estudiado, el 89,7\% de las pacientes presentaban una periodontitis moderada y $10.3 \%$ de las pacientes sufrían de periodontitis leve. Del total de pacientes que padecían de enfermedad periodontal moderada, el $55.5 \%$ proviene de la zona rural. Al interpretar la prueba de hipótesis, esto no fue estadísticamente significativo $\boldsymbol{p}=\mathbf{0 . 8 6 2}$.

Seguido el análisis de los datos y correlacionando las variables, estado civil y enfermedad periodontal previo al tratamiento dental, se determinó que, el $41 \%$ de las pacientes atendidas pertenecían a un estado civil unión de hecho estable; al mismo tiempo, la prevalencia de enfermedad periodontal moderada fue del $41.4 \%$; el $24.1 \%$ de las pacientes eran casadas, de las cuales el $10.3 \%$ tenían periodontitis moderada y el $13.8 \%$ periodontitis moderada. De las pacientes solteras, todas presentaban periodontitis moderada, lo que representó el $34.5 \%$ del total global. Estos datos fueron estadísticamente significativos $(\boldsymbol{p}=\mathbf{0 . 5})$.

Con respecto a la escolaridad, no se encontró correlación estadísticamente significativa entre las variables en estudio. Del total de mujeres estudiadas el 17.2 \% alcanzó la primaria; de las mismas, el 3.4 \% presentó periodontitis leves y el $13.8 \%$, moderada. Las mujeres que alcanzaron la secundaria representó el 69 \% del total global; el $62.1 \%$ tiene periodontitis moderada y solo $6.9 \%$ tiene periodontitis leve. Aquellas mujeres que alcanzaron una carrera profesional (13.8\%) presentaron enfermedad periodontal moderada (13.8\%). Con respecto a la enfermedad periodontal y el parto pretérmino, el 50 \% presentó parto pretérmino y el otro 50 \% no. En la clasificación EP moderada, el 44.4 \% presentó parto pretérmino y el 55.6 \% no lo presentó. De las pacientes sanas (14 pacientes), solo el 7.1 \% (que corresponde a un paciente) presentó PP y el 92.9 \% no presentó el fenómeno de estudio (ver Tabla 1). 


\begin{tabular}{|c|c|c|c|c|c|}
\hline & & & Parto & maturo & Tata \\
\hline & & & Sí & No & Toldil \\
\hline \multirow{6}{*}{$\begin{array}{l}\text { Tipo de enfermedad periodontal } \\
\text { posterior al tratamiento periodontal }\end{array}$} & \multirow{2}{*}{ Leve } & Recuento & 3 & 3 & 6 \\
\hline & & $\%$ & $50,0 \%$ & $50,0 \%$ & $100,0 \%$ \\
\hline & \multirow{2}{*}{ Moderado } & Recuento & 4 & 5 & 9 \\
\hline & & $\%$ & $44,4 \%$ & $55,6 \%$ & $100,0 \%$ \\
\hline & \multirow{2}{*}{ Sano } & Recuento & 1 & 13 & 14 \\
\hline & & $\%$ & $7,1 \%$ & $92,9 \%$ & $100,0 \%$ \\
\hline \multirow{2}{*}{\multicolumn{2}{|c|}{ Total }} & Recuento & 8 & 21 & 29 \\
\hline & & $\%$ & $27,6 \%$ & $72,4 \%$ & $100,0 \%$ \\
\hline
\end{tabular}

Para el peso del bebé a la hora del nacimiento, la media de peso en gramos fue de $3171 \mathrm{~g}$. El $2.5 \%$ de las pacientes con enfermedad periodontal leve no presentaron BPN y el $50 \%$ sí. Las pertenecientes al diagnóstico de periodontitis moderada, el $11.1 \%$ presentó BPN y el 85.7 \% no; el $14.3 \%$ de las pacientes diagnosticadas como sanas sí dieron a luz a niños con BPN; sin embrago, el $79.3 \%$ no dio a luz niños con BPN (ver Tabla 2).

\begin{tabular}{|c|c|c|c|c|c|}
\hline & & & $\begin{array}{r}\text { Bajo } \\
\text { nacer } \mathrm{d}\end{array}$ & $\begin{array}{l}\text { so al } \\
\text { tómico }\end{array}$ & Total \\
\hline & & & Sí & No & \\
\hline \multirow{6}{*}{$\begin{array}{l}\text { Tipo de enfermedad periodontal } \\
\text { posterior al tratamiento periodontal }\end{array}$} & \multirow{2}{*}{ Leve } & Recuento & 3 & 3 & 6 \\
\hline & & $\%$ & $50,0 \%$ & $50,0 \%$ & $100,0 \%$ \\
\hline & \multirow{2}{*}{ Moderado } & Recuento & 1 & 8 & 9 \\
\hline & & $\%$ & $11,1 \%$ & $88,9 \%$ & $100,0 \%$ \\
\hline & \multirow{2}{*}{ Sano } & Recuento & 2 & 12 & 14 \\
\hline & & $\%$ & $14,3 \%$ & $85,7 \%$ & $100,0 \%$ \\
\hline \multirow{2}{*}{\multicolumn{2}{|c|}{ Total }} & Recuento & 6 & 23 & 29 \\
\hline & & $\%$ & $20,7 \%$ & $79,3 \%$ & $100,0 \%$ \\
\hline
\end{tabular}

Posteriormente, se procedió a comprobar los parámetros de las variables dependiente, PP y peso del bebé a la hora del nacimiento. Para comprobar la normalidad de los datos se aplicó la prueba de Shapiro- Wilks, la cual demostró la normalidad de los datos, con un $\boldsymbol{p}=\mathbf{0 . 3 6}$, comparado con el $\boldsymbol{\alpha}=\mathbf{0 . 0 5}$ es no significativo y se asume la hipótesis nula para ambas variables (datos son normales). El Diagnóstico de Homogeneidad de la Varianza de los residuos de los tratamientos mediante la Prueba de Levene, $\boldsymbol{p}=\mathbf{0 . 9 9}$, mayor al nivel crítico de comparación $\boldsymbol{\alpha}=\mathbf{0 . 0 5}$. Este análisis dio "no significativo", lo que demuestra la Homogeneidad de Varianza de residuos. 
El análisis de varianza realizado entre las variables, enfermedad periodontal posterior al tratamiento Vs BPN y PP, determina si existe o no, la relación de causa-efecto de la enfermedad periodontal como causa del bajo peso al nacer y parto pretérmino.

No existe un efecto significativo de la enfermedad periodontal posterior al tratamiento, sobre la variable respuesta bajo peso al nacer y parto pretérmino, lo cual se evidencia con un $\boldsymbol{p}=\mathbf{0 . 8 1}$ y $\boldsymbol{p}=\mathbf{0 . 2 4}$ respectivamente (probabilidad aleatoria del suceso), que resultó ser mayor que el nivel crítico de comparación, $\boldsymbol{\alpha}=\mathbf{0} \mathbf{0 . 5}$. Por tanto, se acepta la hipótesis nula. Esto quiere decir que, la respuesta estadística no es significativa, lo cual demuestra que no existe una relación de causaefecto de la enfermedad periodontal sobre el PP y BPN (ver Tabla 3 y 4).

\begin{tabular}{|l|r|r|r|r|r|}
\hline \multicolumn{1}{|c|}{ Tabla 3. Análisis de la Varianza de la enfermedad periodontal y parto pretérmino } \\
\hline F.V. & \multicolumn{1}{c|}{ SC } & \multicolumn{1}{c|}{ gl } & \multicolumn{1}{c|}{ CM } & \multicolumn{1}{c|}{ F } & p-valor \\
\hline Modelo. & 6,43 & 2 & 3,22 & 1,47 & 0,2474 \\
\hline Enfer-Periodontal-PosTrat & 6,43 & 2 & 3,22 & 1,47 & 0,2474 ns \\
\hline Error & 56,73 & 26 & 2,18 & & \\
\hline Total & 63,16 & 28 & & & \\
\hline
\end{tabular}

\begin{tabular}{|c|c|c|c|c|c|}
\hline F.V. & SC & gl & CM & $\mathbf{F}$ & p-valor \\
\hline Modelo. & 157715,66 & 2 & 78857,83 & 0,21 & 0,8159 \\
\hline Enfer-Periodontal-PosTrat & 157715,66 & 2 & 78857,83 & 0,21 & 0,8159 ns \\
\hline Error & 9995569,65 & 26 & 384444,99 & & \\
\hline Total & 10153285,31 & 28 & & & \\
\hline
\end{tabular}

Para determinar el efecto de la gravedad de la enfermedad periodontal sobre las variables respuestas PP y BPN, se ejecutó comparaciones ortogonales de las diferentes categorías. Se encontró que para el parto pretérmino, el contraste enfermedad periodontal "leve Vs moderado" fue estadísticamente no significativo, al igual que el contraste "moderado Vs Sano", lo que corroboró más, que el parto pretérmino no depende del tipo ni de la usencia de la enfermedad periodontal (ver Tabla 5).

Tabla 5. Tabla que detalla contrastes ortogonales de las categorías de la enfermedad periodontal y parto pretérmino.

\begin{tabular}{|l|c|c|c|c|c|c|c|}
\hline Enfer-Periodontal-PosTrat & Contraste & E.E. & SC & gl & CM & F & p-valor \\
\hline "Leve VS Sano" & 1,08 & 0,72 & 4,89 & 1 & 4,89 & 2,24 & 0,1466 ns \\
\hline "Moderado VS Sano" & 0,82 & 0,63 & 3,71 & 1 & 3,71 & 1,70 & 0,2036 ns \\
\hline Total & 6,43 & 2 & 3,22 & & 1,47 & 0,2474 & \\
\hline
\end{tabular}


Por otro lado, también se realizó el análisis estadístico a través de contrastes ortogonales de las variables enfermedad periodontal Vs bajo peso al nacer. Tras aplicar los contrastes, se obtuvo un $\boldsymbol{p}=\mathbf{0 . 6 7}$, para el contraste "Leve Vs Moderado" y un $\boldsymbol{p}=\mathbf{0 . 7 6}$ para "Moderado Vs Sano", de igual forma se puede apreciar que el tipo ni grado de la enfermedad periodontal es un factor causa para el bajo peso al nacer (ver Tabla 6).

\begin{tabular}{|l|c|c|c|c|c|c|c|}
\hline \multicolumn{2}{|l|}{ Tabla 6. Contrastes de la enfermedad periodontal y bajo peso al nacer } \\
\hline Enfer-Periodontal-PosTrat & Contraste & E.E. & SC & gl & CM & F & p-valor \\
\hline "Leve VS Sano" & 129,76 & 302,55 & 70720,24 & 1 & 70720,24 & 0,18 & 0,6715 ns \\
\hline "Moderado VS Sano" & 79,46 & 264,91 & 34589,42 & 1 & 34589,42 & 0,09 & 0,7666 ns \\
\hline Total & & & 157715,66 & 2 & 78857,83 & 0,21 & 0,8159 \\
\hline
\end{tabular}

\section{DISCUSIÓN}

Muchos estudios como el de Offenbacher, Jared, y O’Reilly, (1998), plantean que los patógenos Gram negativos provenientes de la enfermedad periodontal y la asociación de las endotoxinas y los mediadores inflamatorios maternos, tienen relación y afectan el desarrollo del feto.

La teoría más razonable que sugiere esta relación, es que las infecciones maternas pueden crear una excesiva producción de citoquinas proinflamatorias y prostaglandinas, mismas que han sido establecidas como mediadoras bioquímicas del parto normal (Keelan y otros, 1999). Las observaciones de elevados niveles de Prostaglandina E2 y factor de necrosis tumoral $\boldsymbol{\alpha}(\boldsymbol{F N T} \boldsymbol{\alpha})$ hacen a estos factores constituyentes y reproducibles del parto prematuro y bajo peso al nacer, en infecciones genitourinarias clínicas o subclínicas, como otro tipo de infección de origen desconocido. La posibilidad de que la enfermedad periodontal podría constituir una infección materna remota, y que podría influir adversamente el desarrollo de parto pretérmino y bajo peso al nacer, ya que factores hormonales aumentan la incidencia y severidad de las enfermedades gingivales (Newman, Taikei, Klokkevold y Carranza, 2010).

Si bien, la enfermedad periodontal no es diferente a otro tipo de infección, es probable que la bacterias de la cavidad oral podría contribuir al desarrollo de alteraciones perinatales adversas, dentro de estos patógenos se incluyen aquellos que son oportunistas como Prevotella, Porphyromonus, Bacteroides, y especies de Peptostreptococcus y que también se han detectado en tracto genital inferior y en infecciones pélvicas en forma de vaginosis bacteriana. Esto conduce a pensar y extrapolar que especies microbianas orales podrían estar asociadas y tener un papel en el proceso del parto a través de la activación de procesos inflamatorios.

Goldenberg, Hauth y Andrews (2000) plantean que las vías de diseminación de la infección incluyen, la vía ascendente a través de la vagina y cérvix, la diseminación hematógena, la vía retrógrada desde la cavidad peritoneal y las inoculaciones accidentales durante procesos intrauterinos como la amniocentesis. La vía hematógena es la ruta más común en términos de diseminación bacteriana y ha sido relacionada con los partos pretérmino y bajo peso al nacer (Ercan, y otros, 2013); March of Dimes; PMNCH; Save the Children; WHO (2012). Con el uso de un modelo animal, Collins, Windley, Arnold y Offenbacher (1994) inocularon P. Gingivalis en el tejido subcutáneo de un hámster, encontrando que el peso del feto fue significantemente afectado 
en comparación con el grupo control, al igual que los niveles de $\boldsymbol{F N T} \boldsymbol{\alpha}$ y PGE2 en el sitio de inoculación.

El presente estudio se llevó a cabo en 29 féminas grávidas, agrupadas inicialmente en tratamiento T1, T2, T3 y T4, con el objetivo de aplicar diferentes terapias periodontales para eliminar la enfermedad periodontal, en el caso de que existiese, para posteriormente hacer las comparaciones de los tratamientos experimentales. Sin embargo, estos grupos fueron modificados, ya que no se logró la eliminación completa de la enfermedad en todas las pacientes que conformaban las repeticiones de cada tratamiento, por lo cual, fue necesario reagrupar y aceptar como tratamiento experimental el tipo de periodontitis (leve, moderado, severa) o su ausencia (sano) obtenido de la segunda inspección clínica.

Autores como Kothiwale y Panwar (2011) han reportado una relación directa causa-efecto del estado inflamatorio del tejido gingival y bebés nacidos antes de término y bajos de peso al nacer. Los resultados de esta investigación tienen una dirección opuesta a la planteada por este autor, ya que en su estudio se evalúa el estado inflamatorio de la encía, que podría variar desde una gingivitis a un agrandamiento gingival, al contrario, este estudio valora clínica y específicamente el tipo de enfermedad periodontal. Fue de importancia realizar este tipo de medición ya que esto permitió tener un acercamiento al tipo de periodontitis, así como al agente bacteriano. Estudios como el de Ercan y otros (2013) reflejan una relación de patógenos como el P. Gingivali, F. Nucleatum, T. Foryithia, como patógenos que frecuentemente invaden el líquido amniótico y producen una respuesta inflamatoria que podría llevar a un PP, al mismo tiempo, estos patógenos de la cavidad oral se relacionan con el desarrollo de la periodontitis agresiva localizada, razón por la cual en este estudio se clasificó la periodontitis según su severidad.

Zermeño y otros (2011), en su investigación presentan resultados similares. No encontraron asociación de la periodontitis y parto pretérmino. Es de interés mencionar que, en este estudio se aplicó tratamiento periodontal a las pacientes para hacer mediciones y comparaciones, tratando de evitar el efecto de bacteria sobre el peso y tiempo de nacimiento de los bebés, por lo cual, después de este procedimiento (realizado entre las 20 y 28 semanas), no se presentó ningún caso de periodontitis severa. Dicho diagnóstico se relaciona con la presencia de bacterias, descritas por Ercan, y otros (2013), que podrían causar parto pretérmino y bajo peso al nacer, activando factores citoquinas relacionado con la cadena de eventos para el parto pretérmino propuesta por Loockwood y Kuczynsky (1999) y Gutiérrez y Pareja (2010).

Asociaciones de factores como la edad materna, enfermedades sistémicas, consumo de alcohol o drogas son factores reportados como factores de riesgo para el parto pretérmino y bajo peso al nacer. Este estudio incorporo a 29 mujeres sanas en edades de 18 a 32 años, lo que facilitó el análisis de la enfermedad como causa del parto pretérmino y bajo peso al nacer, ya que se excluyeron variables que podrían causar sesgos en la investigación. El objetivo de este trabajo fue determinar la enfermedad periodontal como causa de BPN y PP, sin embargo, es de importancia ahondar, desde un punto de vista microbiológico, y detallar las vías de contaminación de las bacterias que se relacionan a la periodontitis severa, ya que esta condición, por medio del daño tisular y el aumento de la permeabilidad vascular podría causar diseminación hematógena y ser la causa de los datos reportados en los estudios de Offenbacher, Jared, y O'Reilly (1998), Goldenberg, Hauth, y Andrews (2000), Keelan, y otros (1999). 


\section{CONCLUSIONES}

El 89.7 \% de las pacientes presentaban una periodontitis moderada y el $10.3 \%$ de las pacientes sufrían de periodontitis leve. Del total de pacientes que padecían de enfermedad periodontal tipo moderada, el 55.5 \% provenía de la zona rural. El diagnóstico que prevaleció según el estado civil y nivel académico fue el de periodontitis moderada. Estos hallazgos no fueron estadísticamente significativo y no indican relación de causa-efecto.

Se reportó una frecuencia de 20.7 \% de niños con bajo peso al nacer y de 28 \% de niños nacidos prematuramente. No se evidencia relación causa-efecto significativo para determinar la enfermedad periodontal como factor causal del parto pretérmino y bajo peso al nacer, con un $\boldsymbol{p}=\mathbf{0 . 2 4 7 4}$ y $\boldsymbol{p}=\mathbf{0 . 8 4 5 9}$, respectivamente.

\section{REFERENCIAS BIBLIOGRÁFICAS}

Armitage, G. (1999). Develoment of a classification system for periodontal deases an condition. Ann periodontology, 4(1).

Beck, S., Wojdyla, D., Say, L., Betran, A. P., Merialdi, M., Harris Requejo, J., Van Look, P. F. y otros. (2010). The worldwide incidence of preterm birth: a systematic review of maternal mortality and morbidity. Bulletin World Health Organization, 88, 31-38.

Collins, J., Windley, H., Arnold, R. \& Offenbacher, S. (1994). Effects of Porphyromonas gingivalis infection on inflammatory mediator response in pregnancy outcome in hamsters. Infection and inmmunity, 435661.

Ercan, E., Eratalay, K., Deren, O., Gur, D., Ozyuncu, O., Altun, B., Akincibay, H. y otros. (2013). Evaluation of periodontal pathogens in amniotic fluid and the role of periodontal disease in pre-term birth and low birth weight. Acta Odontologica Scandinavica, 71(3-4), 553-559. Retrieved from http://hinarilogin.research4life. org/uniquesiginformahealthcare.com/ uniquesig0/doi/full/10.3109/00016357.2012 .697576

Goldenberg, R. L., Culhane, J. F., lams, J. D. \& Roberto, R. (2008). Epidemiology and causes of preterm birth. Lancet, 371, 75-84.
Goldenberg, R. \& Tamura, T. (1996). Prepregnancy weight and pregnancy outcome. The journal of the American Medical Association, 275, 1127-28.

Goldenberg, R., Hauth, J. \& Andrews, W. (2000). Intrauterine infection and preterm delivery. N Englan Journal of Medicine, 1500-07.

Gutiérrez, F. R. \& Pareja, M. V. (2010). parto prematuro y bajo peso al nacer y su relación con la enfermedad periodontal. Revista Kiru, 7(1), 43-49.

Keelan, J., Marvin, K., Sato, T., Coleman, McCowan, L. \& Mitchell, M. (1999). Cytokine abundance in placental tissues: evidence of inflammatory activation in gestational membranes with term and preterm parturition. American Journal of Obstetric and Ginecology., 181, 1530-6.

Kothiwale, S. \& Panwar, V. (2011). Poor periodontal health of pregnant women as a risk factor for preterm and low birth weight. Indian Journal of Dentistry, 2(4), 129-33. Retrieved from http:// ac.els-cdn.com/S0975962X11600324/1s2.0-S0975962×11600324-main. pdf?_tid=e2f2a0ae-62bf-11e4-8a 4d00000 aab0fO2 \&acdnat $=1414953881$ f8cec5a9504c7d3d76b42c1e0167421a 
Loockwood, C. \& Kuczynsky, E. (1999). Markers of riskfor preterm delivery. Jornal of Perinatal Medicine, 27, 5-20.

March of Dimes; PMNCH; Save the Children; WHO. (2012). Born Too Soon: The Global Action Report on Preterm Birth. (Howson, M. Kinney \& J. Lawn., Eds.) World Health Organization.

Newman, M. G., Taikei, H. H., Klokkevold, P. R. \& Carranza, F. A. (2010). Periodontología Clínica. (10 ed.). Mexico: Mc Graw Hill.

Newman, M. H., Klokkevold, P. R. \& Carranza, F. (2014). Carranza's Clinical Periodontology (Vol. 11). Riverport lane, St. Louis, Missouri: Saunders.
Offenbacher, S., Jared, H, \& O'Reilly, P. (1998). Potential pathogenic mechanisms of periodontitis associated pregnancy complications. Annales of Periodontology, 233-250.

Ramseier. (2010). Periodontology online. School of Dental Medicine, University of Bern, Deparmen of Periodontology. Retrieved from www.periodontalchart-online.com

Zermeño, N. J., Flores, A. C., Saldívar, R. D., Soria, L. J., Garza, R. M, \& Iglesias, B. L. (2011). Enfermedad periodontal como factor de riesgo para presentar resultados perinatales adversos. Revista Chilena obstétrica ginecológica, 76(5), 338-343. 